\title{
Lattice-free models of directed cell motility
}

\author{
Carolyn Irons \\ School of Mathematics and Statistics, University of Canterbury, Christchurch \\ 8140, New Zealand. \\ Michael J. Plank* \\ School of Mathematics and Statistics, University of Canterbury, Christchurch \\ 8140, New Zealand. \\ Te Pūnaha Matatini, a New Zealand Centre of Research Excellence. \\ Matthew J. Simpson \\ Mathematical Sciences, Queensland University of Technology (QUT), GPO Box \\ 2434, Brisbane, Queensland 4001, Australia. \\ Tissue Repair and Regeneration Program, Institute of Health and Biomedical \\ Innovation, QUT, Brisbane, Queensland, Australia.
}

\begin{abstract}
Directed cell migration often occurs when individual cells move in response to an external chemical stimulus. Cells can respond by moving in either the direction of increasing (chemoattraction) or decreasing (chemorepulsion) concentration. Many previous models of directed cell migration use a lattice-based framework where agents undergo a lattice-based random walk and the direction of nearest-neighbour motility events is biased in a preferred direction. Such lattice-based models can lead to unrealistic configurations of agents, since the agents always move on an artificial lattice structure which is never observed experimentally. We present a lattice-free model of directed cell migration that incorporates two key features. First, agents move on a continuous domain, with the possibility that there is some preferred direction of motion. Second, to be consistent with experimental observations, we enforce a crowding mechanism so that motility events that would lead to agent overlap are not permitted. We compare simulation data from the new lattice-free model with a more traditional lattice-based model. To provide additional insight into the lattice-free model, we construct an approximate conservation statement which corresponds to a nonlinear advection-diffusion equation in the continuum limit. The solution of this mean-field model compares well with averaged data from the individual-based model.
\end{abstract}

Key words: advection; chemotaxis; biased random walk; exclusion process; nonlinear diffusion; scratch assay.

Preprint submitted to Physica A 1 July 2015 
* Corresponding author

Email address: michael.plank@canterbury.ac.nz, Telephone +64 3 3642699 (Michael J. Plank). 


\section{Introduction}

2 Directed cell movement is essential for a variety of physiological processes including wound healing, angiogenesis, axons guidance and bacterial migration

${ }_{4}[1-5]$. Typically, cells move in a particular direction in response to an external factor, such as a chemical stimulus. For example, white blood cells can move

6 towards a site of infection in response to chemicals released by the bacteria causing the infection $[5,6]$. The directed movement of cells along a chemical

8 gradient is called chemotaxis. A chemical which acts as an attractant is called a chemoattractant, whereas a chemical which acts as a repellent is called a chemorepellent [5].

Cell motility is often studied using in vitro techniques, such as a scratch as12 say, where cells are grown in a two-dimensional monolayer before part of the population is scratched, leaving a region of unoccupied substrate that the 14 remaining cells subsequently recolonize [7]. Other experiments are used to investigate chemotaxis specifically by, for example, investigating the relationship bias cells exhibit [5].

Discrete random walk models are often used to study collective cell motion, including chemotaxis $[1,2,8]$. These models produce snapshots of the spreading population and movie-based data that are easy to compare with experimental images and time-lapse data [9]. There are two key classes of random walk model that have been used to represent collective cell migration processes.

Lattice-based random walk models represent the spatial domain as a regular

lattice. Individual cell motility events are usually modelled using a nearestneighbour random walk process. Many relevant applications of collective cell spreading involve situations where interactions between neighbouring cells are important since experiments are often initiated at a relatively high cell density [10-12]. Experimental observations of the effects of cell-to-cell interactions [12] have motivated the development of random walk models which incorporate crowding effects, For example, in an exclusion process [13], each lattice site can be occupied by, at most, a single agent. In this type of model, individual movement events depend on the state of the system. For example, a motility event that would place an agent on an occupied site would be aborted and these aborted events are interpreted as a crowding effect [14-16]. Directional bias can be incorporated into these models by allowing the probabilities of choosing a target site for the nearest-neighbour random walk to be unequal $[1,17]$, although other models of directed motion are also possible [18,19]. Latticebased exclusion process models have been used to represent many processes in cell biology, including cancer cell migration [7,14], wound healing $[20,21]$ and development [9]. In a lattice-based model, the direction of movement is 
chosen from a discrete set of directions corresponding to nearest-neighbour

42 lattice sites, for example: left, right, up or down on a two-dimensional square lattice.

Images from experimental investigations clearly show that individual cells are not arranged on a regular lattice $[7,12,22]$. Lattice-free random walk models permit agents to reside within a continuous spatial domain and allow direction of movement to be a continuous variable [24]. Continuum limit approximations have been derived for a population of cells undergoing a biased position-jump process $[23,25]$ or biased velocity-jump process [26], and can be either a chemotaxis equation or an anisotropic diffusion equation, depending on the strength of the bias [27]. However, these earlier results do not include cell-to-cell interactions and crowding effects, which are thought to have a major impact on collective behaviour [2]. More recently, cell-to-cell interactions have been incorporated into lattice-free models using various individual-level mechanisms. For example, refs. $[28,29]$ used a simple, unbiased random walk with an attempt-and-abort volume exclusion mechanism; this has been extended to the biased case [30]. Refs. [31,32] used Brownian motion plus drift to model agent motility with a hard disk collision mechanism for volume exclusion. Refs. [33,34] modelled crowding using a neighbour-dependent interaction force, rather than a strict volume exclusion mechanism. These models could include a global bias, as well as local neighbour-dependent bias, but the results presented applied to the case without global bias. The different individual-level mechanisms of $[28,30,31,33,34]$ give rise to different nonlinear advection-diffusion equations or integro-differential equations for the average agent density.

${ }_{66}$ There has been an increasing interest in deriving approximate mean-field (continuum-limit) descriptions of individual-based random walk models with cell-to-cell interactions. These often take the form of a partial differential equation (PDE) for agent density. Such descriptions can provide greater insight than is possible from simulations of an individual-based model alone. For example, the averaged behaviour of an unbiased lattice-based exclusion process can be described by the linear diffusion equation [13], whereas combining proliferation with unbiased motility in a lattice-based model leads to a reaction-diffusion PDE which is a generalization of the Fisher-Kolmogorov equation [35-37]. Incorporating directional bias in a lattice-based exclusion process leads to a nonlinear advection-diffusion PDE [17]. Contact effects, such as cell-to-cell adhesion, can lead to a nonlinear diffusion equation [14,38,39], a nonlinear advection equation [40] or an equation of Cahn-Hilliard type [41,42]. However, the form of the nonlinearity can depend on the geometry of the lattice (e.g. square or triangular) [43], highlighting the fact that the choice of lattice is non-unique and can affect model predictions.

${ }_{82}$ One of the key differences between lattice-based and lattice-free models is in 
the maximum density of agents. The highest density arrangement of circles in a

plane is a hexagonal tessellation, giving an area occupancy of $\pi / \sqrt{12}$, which is greater than the area occupancy associated with a square lattice arrangement, $\pi / 4$. However, random variations in agent locations mean that the lattice-free model is extremely unlikely to get close to its theoretical maximum density, whereas in a lattice-based model the agents are always arranged in a regular pattern, making it much easier to achieve the maximum density [28]. Because of this difference, cell proliferation in a lattice-free model leads to a source term in the PDE that is smaller than the corresponding source term in a latticebased PDE description [44]. Another consequence of this difference is that a greater proportion of attempted motility events are aborted in a lattice-free model than a corresponding lattice-based model. These differences manifest in the mean-field PDE description since an unbiased lattice-based model leads to a linear diffusion PDE whereas an unbiased lattice-free model leads to a nonlinear diffusion PDE [45]. However, the appropriate mean-field PDE description of a lattice-free model incorporating crowding effects of directional bias have not yet been considered or compared with the equivalent results from a lattice-based model.

In this work we present and analyze a lattice-free model of biased cell motility that is an extension of previous models of unbiased cell motility [28,45]. We model directional bias at the individual agent level using a continuous circular distribution of movement directions that is peaked in a particular direction. This is different to the mechanism of $[31,32]$, which was based on background convective drift, and [30], which was effectively a piecewise constant distribution of movement directions. Circular distributions have been used previously to model directed movement in both position-jump processes $[23,25,46]$ and velocity-jump processes $[23,26,27,47]$, but these studies do not include cell-tocell interactions. We do not include cell proliferation or cell death since we wish to focus on the impact of directional bias in the motility mechanism. Neglecting cell proliferation is reasonable since the timescale of cell motility is approximately 20-30 minutes, whereas cell proliferation occurs over much larger time scales, approximately 24-48 hours [48]. The neglect of cell proliferation and death means that our model is relevant for in vitro experiments which are typically conducted over short intervals of time, typically less than 24 hours, where an excess of nutrients is supplied so that cell death does not occur [48]. We derive an approximate mean-field nonlinear advection-diffusion PDE for the lattice-free model and show that this description differs from the corresponding PDE description of a lattice-based model. We compare solutions of the lattice-free PDE to averaged density profiles from simulations of the individual-based model, as well as comparing lattice-free results with an analogous model based on a square lattice [17]. Although other lattice geometries are possible, such as a triangular lattice [14,49] or an irregular lattice $[50,51]$, we choose to use a square lattice for comparison as this is the most commonly used lattice geometry in models of collective cell behaviour 
$[1,7,16,21,52-56]$. Consistent with previous findings $[28,44]$, we show that the ${ }_{128}$ lattice-based and lattice-free models behave similarly when the agent density is sufficiently low, but behave differently when the agent density is high.

\section{Individual-based model}

We consider a population of $N$ agents moving in a rectangular region, $-a \leq$ $32 x \leq a,-b \leq y \leq b$. Since we do not consider proliferation or death, the total population size $N$ is fixed. All agents are disks of diameter $\Delta$.

\subsection{Lattice-free model}

We consider a discrete time process where, during each time step of duration $\tau$, each agent attempts to move with probability $P_{m}$. The direction of each potential movement event is a random variable $\Theta$, with probability density function $f(\theta)$. The agent attempts to move in a straight line from the current location, $(x, y)$, to a target location

$$
(x+\Delta \cos (\Theta), y+\Delta \sin (\Theta)) \text {. }
$$

If this straight-line path would cause the agent attempting to move to overlap with another agent, at any point between the initial location and the target location, the potential movement event is aborted and the agent remains at $(x, y)$. This is a simple volume exclusion model that treats the cells as disks of fixed diameter [28]. Other types of crowding mechanism have also been considered, for example a hard sphere collision model [31,57], or a repulsion force between neighbouring cells that is a function of the distance between them, representing the ability of cells to deform as a result of cell-cell contacts [33]. We choose a fixed step length $\Delta$, which coincides with the agent diameter, and this choice is consistent with the lattice-based model (Sec. 2.2); however, it is straightforward to generalise this and allow the step length to be drawn from a distribution [19]. The distribution of movement directions is determined by $f(\theta)$. If $f(\theta)$ is a uniform distribution on $[0,2 \pi]$, the movement is unbiased (Fig. $1(\mathrm{a})-(\mathrm{b}))$ and the model is equivalent to that of [28]. If $f(\theta)$ is peaked around some angle $\theta_{0}$ then the movement is biased in the direction of $\theta_{0}$ (Fig. 1(c)-(d)). The fact that we treat $\theta$ as a continuous variable, rather than being chosen from a discrete set of directions, makes the lattice-free model fundamentally different from a lattice-based model. For example, even we were to initialise the agents regularly, this regularity would be lost as the lattice-free model evolves because of the continuous range of possible movement directions. 
(a)

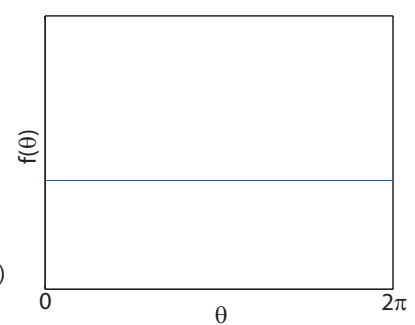

(c)

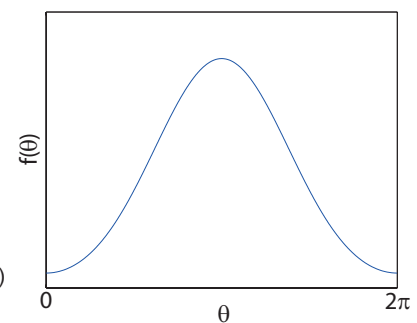

(b)
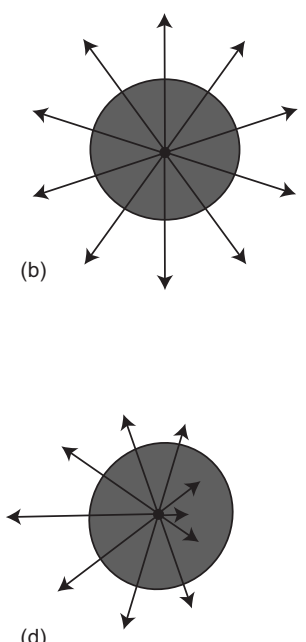

width $\Delta$

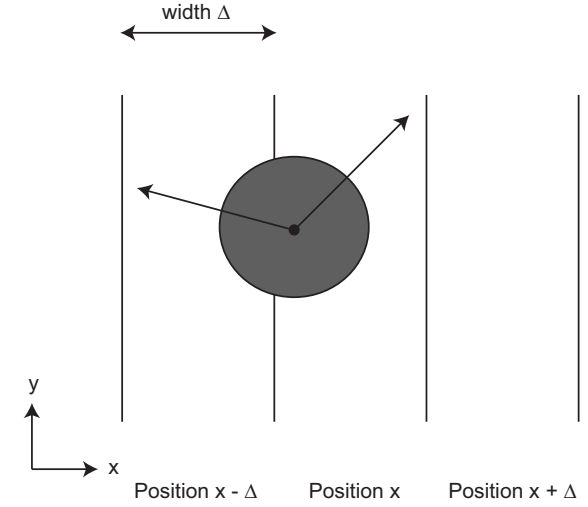

(e)

Fig. 1. Schematic illustration of the lattice-free model. (a) Shows $f(\theta)$ in the case where there is no preferred direction and all directions are equally likely, as illustrated in (b). (c) Shows $f(\theta)$ in the case where the probability density function is peaked at a preferred angle $\theta_{0}=\pi$, meaning that movement is more likely to occur in that direction, as illustrated in (d). The schematic in (e) shows how the spatial domain can be discretized into strips of width $\Delta$ to facilitate the construction of an approximate conservation statement that allows us to derive an approximate PDE description of the lattice-free model with directional bias.

\subsection{Lattice-based model}

We consider a lattice-based model that has been studied previously [17] and we only describe it briefly here. The lattice-based model is an exclusion process on a square lattice, with lattice spacing $\Delta$. During each time step, of duration $\tau$, each agent is given the opportunity to move with probability $P_{m}$. A motile agent at site $(x, y)$ attempts to move to $(x \pm \Delta, y)$ or $(x, y \pm \Delta)$, with probability $\left(1 \pm \rho_{x}\right) / 4$ or $\left(1 \pm \rho_{y}\right) / 4$, respectively. The parameters $\rho_{x} \in[-1,1]$ and $\rho_{y} \in$ $[-1,1]$ control the direction and strength of the bias: if $\rho_{x}=\rho_{y}=0$, the movement in unbiased; if $\rho_{x}=1$, the horizontal component of all successful motility events will be directed in the positive $x$ direction whereas if $\rho_{x}=-1$ the horizontal component of all successful motility events will be directed in the negative $x$ direction. Since the model is an exclusion process any potential motility event that would place an agent on an occupied site is aborted.

\subsection{Simulation method}

Each realisation of the individual-based model is initialised with $N$ agents placed uniformly, at random, within the region $-x_{0}<x<x_{0}$ and $-b<y<b$. 
In the lattice-based model, no more than one agent is permitted to occupy any lattice site. In the lattice-free model, no two agents are permitted to overlap. A potential location $\left(x_{i}, y_{i}\right)$ is generated for the $i^{\text {th }}$ agent and the distance between $\left(x_{i}, y_{i}\right)$ and $\left(x_{j}, y_{j}\right)$ is calculated for $j=1, \ldots, i-1$. If this distance is less than $\Delta$ for any $j$, the potential location $\left(x_{i}, y_{i}\right)$ is discarded and a new location is generated. This process is repeated until an allowable location for the $i^{\text {th }}$ agent is found, and further repeated until $N$ agents have been placed within the region $-x_{0}<x<x_{0}$ and $-b<y<b$.

The algorithm for initialising agent locations is a random sequential adsorption process. Agents placed later on in such a process tend to be more jammed in than agents placer earlier [58] and there is a maximum achievable density of agents, which is approximately that of a lattice that is $70 \%$ occupied [59]. To avoid problems associated with approaching the maximum density, we set the initial number of agents to correspond to a lattice that is $60 \%$ occupied in the region $-x_{0}<x<x_{0}$ and $-b<y<b$ in both models. This means that each simulation is initialized with $N=0.6\left(4 x_{0} b\right)$ agents. Other methods for initialising agent locations in the lattice-free model are possible, for example initialising agents on a regular lattice and then adding noise.

For both the lattice-based and lattice-free models, each agent is given the opportunity to move, one at a time, with probability $P_{m}$ per time step. Any potential motility event that would place an agent outside the domain, $-a \leq$ $x \leq a,-b \leq y \leq b$, is aborted. The horizontal length of the domain, $2 a$, is chosen to be sufficiently large that agents do not typically reach the boundaries at $x= \pm a$ during the time scale of the simulation.

In the lattice-free model, we use a wrapped normal distribution for the direction of movement, which is a circular distribution defined on $[0,2 \pi][60]$. In practice, we generate random deviates from a normal distribution on $\mathbb{R}$, with probability density function

$$
f(\theta)=\frac{1}{\sigma_{d} \sqrt{2 \pi}} e^{-\frac{\left(\theta-\theta_{0}\right)^{2}}{2 \sigma_{d}^{2}}}, \quad \theta \in \mathbb{R} .
$$

These random deviates are subsequently inserted into trigonometric functions in Eq. (1), so this is equivalent to generating random deviates from a wrapped normal distribution. The parameter $\sigma_{d}$ controls the strength of the bias: when $\sigma_{d}$ is large, the wrapped normal distribution approximates a uniform distribution on $[0,2 \pi]$ (see Fig. 1(a)-(b)); as $\sigma_{d}$ becomes smaller, the distribution becomes increasingly peaked at $\theta=\theta_{0}$, corresponding to stronger bias (see Fig. 1(c)-(d)). To simplify our comparison of the lattice-based and lattice-free frameworks we set $\rho_{y}=0$ in the lattice-based model and $\theta_{0}=\pi$ in the latticefree model so the direction of bias is always in the negative $x$ direction. This 
is for convenience only and other choices of bias direction are equally possible.

Our choices of geometry and initial condition mean that the distribution of agents is uniform in the vertical direction. Although both individual-based models are genuinely two-dimensional process, by averaging in the vertical direction, we can study the dynamics of the models in a one-dimensional framework $[9,28]$. To obtain average agent density profiles, we perform $m$ identically prepared realisations of the individual-based model. In the $k^{\text {th }}$ realisation, we count the number of agents $n_{k}(x, t)$ whose horizontal coordinate lies in the interval $[x-\Delta / 2, x+\Delta / 2$ ) at time $t$ (see Fig. 1(e)). The average agent density $C(x, t)$ is calculated by averaging over the $m$ realisations to give

$$
C(x, t)=\frac{\Delta}{2 b m} \sum_{i=1}^{m} n_{i}(x, t) .
$$

This is a double average over the vertical coordinate $y$ and over the $m$ realisations. Increasing either $m$ or $b$ increases the number of agents used to estimate $C(x, t)$. The factor $\Delta /(2 b)$ normalises the agent density so that $C(x, t)$ is dimensionless and $C(x, t)=1$ corresponds to the density of a fully occupied square lattice of lattice spacing $\Delta$. The initial condition described above is equivalent to $C(x, 0)=0.6$ for $-x_{0} \leq x \leq x_{0}$. Note that $C$ is equal to $4 \phi / \pi$ where $\phi$ is the fraction of area occupied. It is therefore possible for $C(x, t)$ to exceed 1 in the lattice-free model (the theoretical upper bound corresponding to a hexagonal arrangement of agents is $\phi=\pi / \sqrt{12}$, i.e. $C=2 / \sqrt{3}$ ). However, it is extremely unlikely for agents to become arranged in such a regular way and previous results [28] show that, even in models where proliferation is included, $C(x, t)$ does not exceed 1 on physiologically relevant timescales.

\section{Mean-field description}

To obtain an approximate mean-field description of the lattice-free model, we divide the domain into vertical strips of width $\Delta$ and we let $C(x, t)$ represent the density of agents in a strip whose centre has horizontal coordinate $x$ (see Fig. 1(e)). To incorporate crowding effects, we assume that an attempted motility event that would place an agent onto a vertical strip occupied at density $C$ is successful with probability given by the function $P(C)$ [45]. Thus $P(C)$ is the conditional probability of a movement being successful given that the movement is attempted. It is reasonable to assume that this function is independent of the bias strength because bias affects the choice of direction but, once that direction is chosen, the likelihood of successful movement only depends the density of agents in that direction. The choice of this function can include various model scenarios. If there are no crowding effects, all potential 
movement attempts are successful, and $P(C) \equiv 1$. In a lattice-based model, if we neglect correlations between the occupancies of agents on the lattice, we have $P(C)=1-C[14,17,43]$. In a lattice-free model, invoking similar assumptions that amount to the neglect of spatial correlations, we have previously shown that $P(C) \approx 1-2 C+C^{2}$ [45]. In all cases, we have $P(0)=1$ so that, in the low-density limit, all attempted movement events are successful. Since $1-2 C+C^{2}=(1-C)^{2}<1-C$ for $0<C<1$, we expect that crowding effects will be stronger in the lattice-free model than in the lattice-based model, which is consistent with previous observations for unbiased motion $[28,44]$.

We now develop an approximate conservation statement for a vertical strip as illustrated in Fig. 1(e), during some time interval of duration $\tau$. We let $r$ and $l$ be the probabilities that an attempted movement event will take an agent initially located in a strip centred at $x$ to a strip centred at $x \pm \Delta$ respectively. The expected change in the density of a strip centred at $x$ during a single time step of duration $\tau$ is

$$
\begin{aligned}
\delta C(x, t)= & P_{m} \tau[r C(x-\Delta, t) P(C(x, t))+l C(x+\Delta, t) P(C(x, t)) \\
& -r C(x, t) P(C(x+\Delta, t))-l C(x, t) P(C(x-\Delta, t))] .
\end{aligned}
$$

Each term in this discrete conservation statement can be interpreted as the probability of an agent in a particular strip attempting to move to a neighbouring strip, multiplied by the probability that such a movement attempt will be successful. In doing this, we are making the standard assumption that the occupancies of adjacent strips are independent [17]. This assumption may not always be valid as short-range correlations in agent can develop under certain situations $[61,62]$. However, this assumption has proved reasonable for unbiased motion, provided agent proliferation is rare relative to agent motility [28]. To obtain a continuous description, we expand $C(x \pm \Delta)$ in a Taylor series about $x$ :

$$
C(x \pm \Delta, t)=C(x, t) \pm \Delta \frac{\partial C(x, t)}{\partial x}+\frac{\Delta^{2}}{2} \frac{\partial^{2} C(x, t)}{\partial x^{2}}+O\left(\Delta^{3}\right)
$$

We now take the continuum limit $\Delta, \tau \rightarrow 0$, with $\Delta^{2} / \tau$ held constant, giving the following PDE for $C(x, t)$ :

$$
\frac{\partial C}{\partial t}=-\frac{\partial}{\partial x}(v(C) C)+\frac{\partial}{\partial x}\left(D(C) \frac{\partial C}{\partial x}\right),
$$

where 


$$
v(C)=\frac{P_{m}(r-l) \Delta}{\tau} P(C), \quad D(C)=\frac{P_{m}(r+l) \Delta^{2}}{2 \tau}\left(P(C)-C \frac{d P}{d C}\right)
$$

In the unbiased case $(r=l)$, we have $v(C) \equiv 0$, leaving a nonlinear diffusion PDE, which is consistent with previous results [45]. In the low-density limit $(C \rightarrow 0, P(C) \rightarrow 1)$, the mean-field PDE reduces to the usual linear advection-diffusion equation [19] since $v(C)$ and $D(C)$ are constants. Equations (3) and (4) can be applied in the lattice-based and lattice-free frameworks. The differences between these models lie in the expression for the probability of successful movement $P(C)$ and the probabilities of moving to a strip to the right $(r)$ or left $(l)$ of the current strip.

In the lattice-based framework, $r$ and $l$ are obtained directly from the individualbased model as $r=\left(1+\rho_{x}\right) / 4$ and $l=\left(1-\rho_{x}\right) / 4$, giving a straightforward correspondence between $\rho_{x}$ and the advection and diffusion coefficients in Eq. (4). As usual with biased random walks, to obtain a well defined PDE description, $P_{m}(r-l) \Delta / \tau$ must remain finite and non-zero in the continuum limit, which implies that $\rho_{x}$ must scale with $\Delta$ as $\Delta, \tau \rightarrow 0[19,23,63]$. For this reason, the mean-field PDE may be inappropriate when the bias is sufficiently strong [25].

In the lattice-free model, $r$ and $l$ depend on the direction distribution $f(\theta)$ more subtly than in the lattice-based model. We calculate the advection and diffusion coefficients in the low-density limit by considering the behaviour of a single, isolated agent that is not subject to any crowding effects. The low-density advection coefficient, $v(0)$, corresponds to the mean horizontal displacement of such an agent per unit time [19]. The horizontal displacement of an isolated, motile agent in a single time step is $\Delta \cos (\Theta)$, so we have

$$
v(0)=\frac{P_{m} E[\Delta \cos (\Theta)]}{\tau}=\frac{P_{m} c_{1} \Delta}{\tau},
$$

where $c_{k}$ is the $k^{\text {th }}$ moment of the distribution of $\cos (\Theta)$ :

$$
c_{k}=E\left(\cos ^{k}(\Theta)\right)=\int_{-\infty}^{\infty} \cos ^{k}(\theta) f(\theta) \mathrm{d} \theta . \quad k=1,2, \ldots
$$

Similarly, the low-density diffusion coefficient, $D(0)$, is equal to half of the mean squared horizontal displacement per unit time [19]:

$$
D(0)=\frac{P_{m} E\left[(\Delta \cos (\Theta))^{2}\right]}{2 \tau}=\frac{P_{m} c_{2} \Delta^{2}}{2 \tau}
$$


Combining these low-density limits with Eq. (4) gives expressions for $v(C)$ and $D(C)$ in the lattice-free model in terms of $P(C)$ and $f(\theta)$ :

$$
\begin{aligned}
v(C) & =\frac{P_{m} c_{1} \Delta}{\tau} P(C), \\
D(C) & =\frac{P_{m} c_{2} \Delta^{2}}{2 \tau}\left(P(C)-C \frac{d P}{d C}\right) .
\end{aligned}
$$

For the wrapped normal distribution in Eq. (2) with preferred direction $\theta_{0}=\pi$, Eq. (5) gives

$$
c_{1}=-e^{-\sigma_{d}^{2} / 2}, \quad c_{2}=\frac{e^{-2 \sigma_{d}^{2}+1}}{2} .
$$

To satisfy the requirement that $c_{1}$ is proportional to $\Delta$ in the continuum limit, the direction distribution $f(\theta)$ needs to approach a uniform distribution (i.e. become increasingly weakly biased) as $\Delta, \tau \rightarrow 0$. As for the lattice-based model, we anticipate that the mean-field PDE will be less accurate for strongly biased motion (i.e. when $\left|c_{1}\right|$ is sufficiently large).

To match the individual-based model, the initial condition for Eq. (3) is

$$
C(x, 0)=\left\{\begin{array}{l}
0.6,-x_{0}<x<x_{0} \\
0, \quad \text { otherwise }
\end{array}\right.
$$

No-flux boundary conditions are applied at $x= \pm a$. Numerical solutions of Eq. (3) are obtained using MATLAB' ${ }^{\circledR}$ pdepe routine, which uses the method of lines [64] and the ode15s ODE routine [65], on a uniformly discretized mesh with spacing $\delta x=0.1$. For the lattice-based model we set $P(C)=1-C$ [17] and for the lattice-free model we set $P(C)=1-2 C+C^{2}[45]$.

\section{Results}

Snapshots from both the lattice-based and lattice-free models are shown in Fig. 2. The effect of the directional bias is clear in both models, causing a drift of agents in the negative $x$ direction.

Averaged agent density profiles are shown in Fig. 3, together with the numerical solution of the corresponding mean-field PDE. In the unbiased case (Fig. 


\section{Lattice-based}

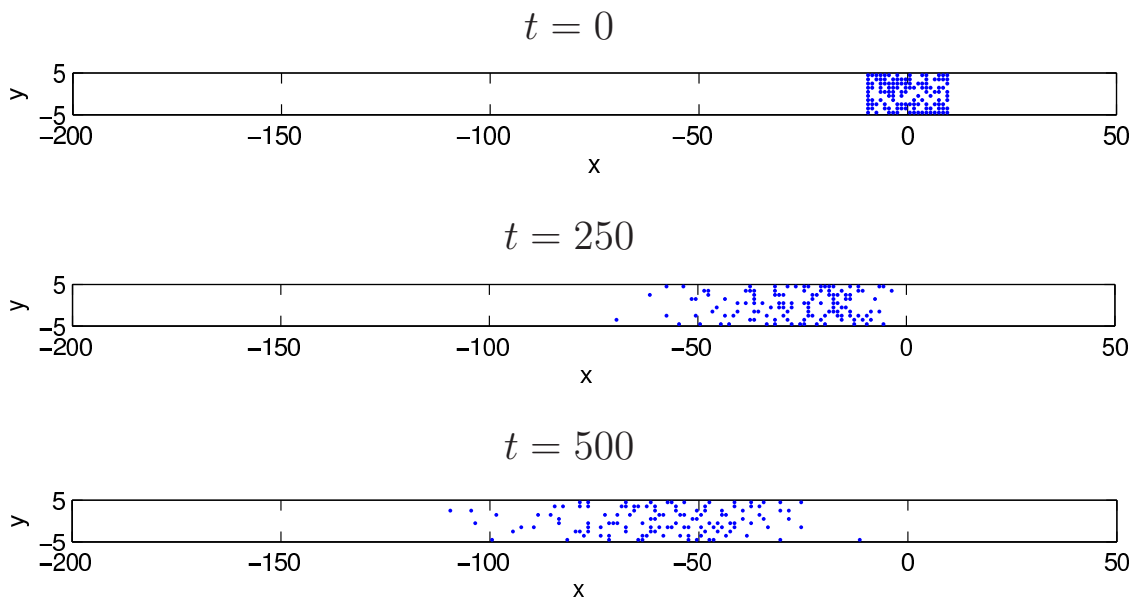

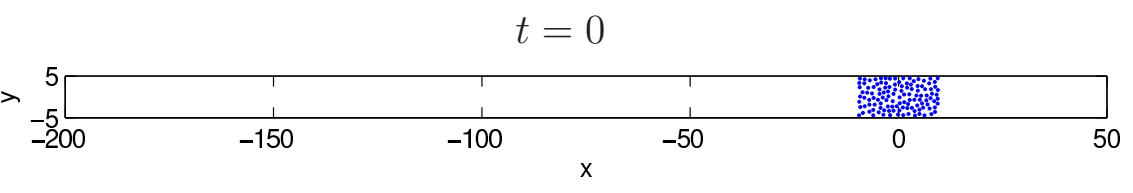
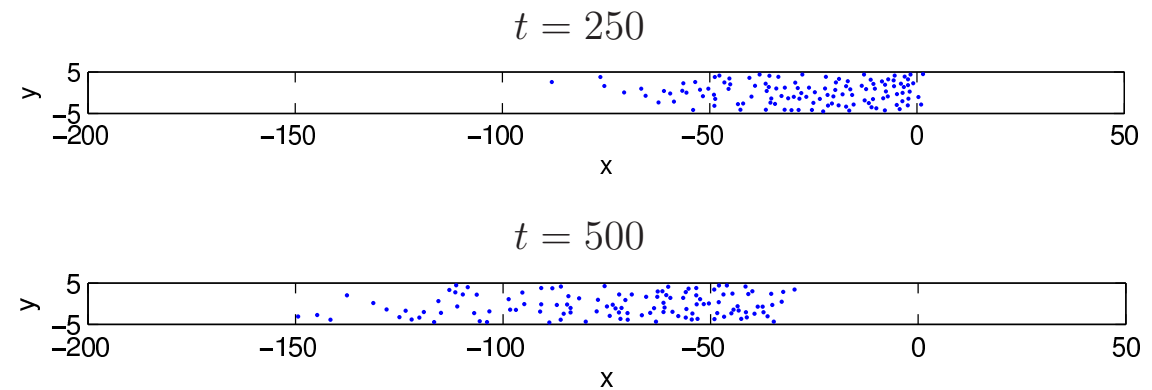

Fig. 2. Snapshots of the lattice-based and lattice-free individual-based models. Parameter values: $P_{m}=1, \rho_{x}=-0.3, \sigma_{d}=1.63$, $\Delta=\tau=1$. Initial condition: $N=120$ agents randomly located in $-10<x<10,-5<y<5$. 


\section{Lattice-based}

(a) Unbiased $\rho_{x}=0$

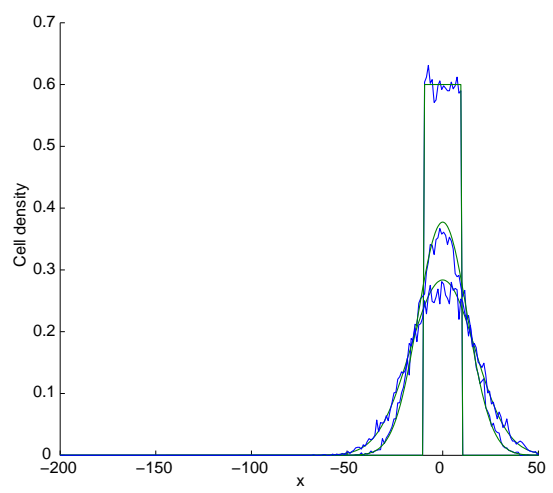

(c) Weak bias $\rho_{x}=-0.15$

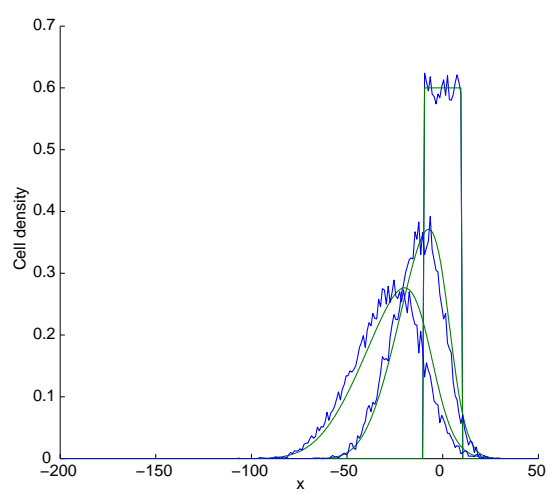

(e) Strong bias $\rho_{x}=-0.3$

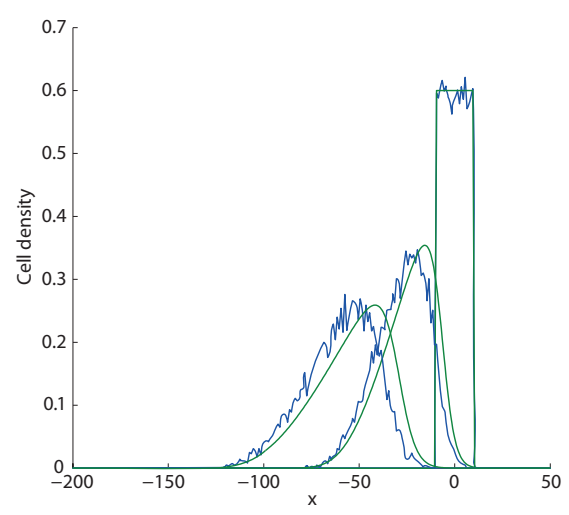

Lattice-free

(b) Unbiased $\sigma_{d} \rightarrow \infty$

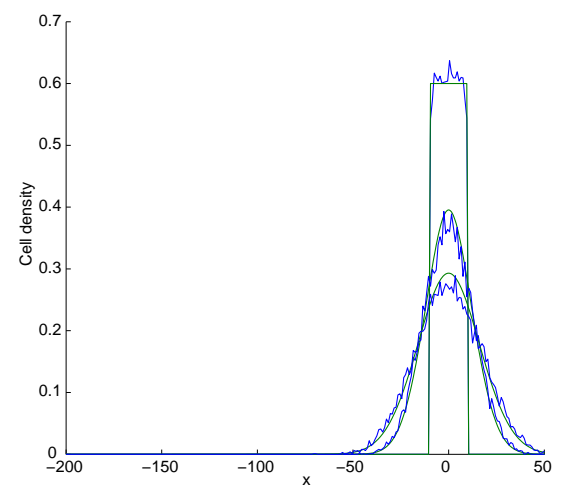

(d) Weak bias $\sigma_{d}=2.4$

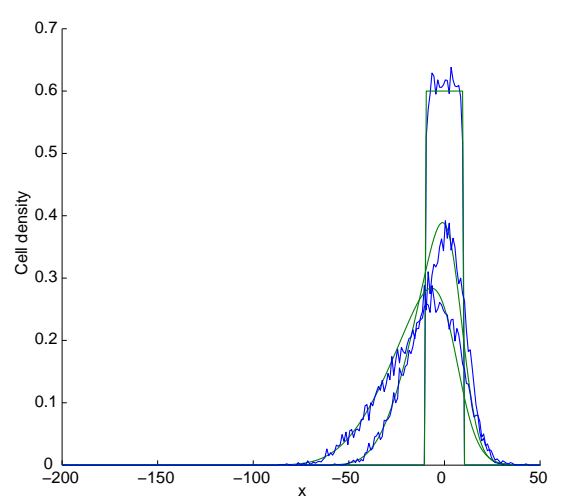

(f) Strong bias $\sigma_{d}=1.8$

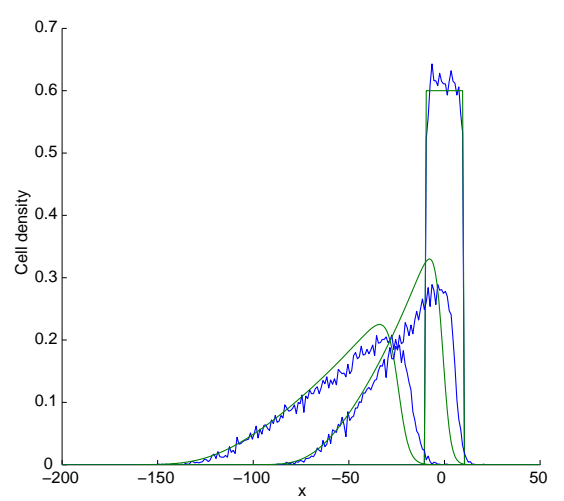

Fig. 3. Average agent density profiles from $m=100$ realisations of the individual-based model (blue) together with solutions of the corresponding mean-field PDE (green) at time $t=0, t=250$ and $t=500$. In the unbiased lattice-free model shown in (b), the direction of movement is chosen from a uniform distribution, i.e. $f(\theta)=1 /(2 \pi)$. Parameter values: $P_{m}=1, \Delta=\tau=1$. Initial condition: $N=120$ agents randomly located in $-10<x<10,-5<y<5$. 
3(a)-(b)), agents move without directional bias and the mean-field PDE is a 324 nonlinear diffusion equation $(v(C) \equiv 0)$. The average agent density spreads symmetrically outwards from the initial condition. When directional bias is introduced (Fig. 3(c)-(f)), the agent density profiles drift in the negative $x$ direction. As expected, the drift is higher when the bias is strong (Fig. 3(e)-(f)) than when the bias is weak (Fig. 3(c)-(d)).

The agent density profiles for biased movement become skewed to the left over

time. This is in contrast to a linear advection-diffusion equation (which describes biased motility without crowding effects), for which the density profiles remain symmetric for all time [19]. The asymmetry develops because agents at the leading edge of the population, where $C(x, t) \approx 0$, are free to move in the direction of the bias without being crowded. These uncrowded agents can traverse large distances relatively quickly. Conversely, agents further back from the leading edge, where $C(x, t) \gg 0$, are more likely to experience aborted motility events due to crowding. This means that the trailing edge of the population moves more slowly than the leading edge. In general, this effect occurs in both the lattice-based and lattice-free models and is more pronounced when the bias is strong. The mean-field PDE predicts the asymmetry quite accurately when bias is weak to moderate, but, as expected, loses accuracy when the bias is strong.

Results in Fig. 4 show the mean horizontal location of all agents, starting from the same initial condition as in Fig. 3 , at time $t=500$, as a function of the bias strength. The mean location in the individual-based model is averaged across all agents in the population and across many identically prepared realizations of the same stochastic process. The solution of the PDE also provides a prediction of mean location [19]

$$
\text { mean location }=\frac{\int_{-a}^{a} x C(x, t) \mathrm{d} x}{\int_{-a}^{a} C(x, t) \mathrm{d} x},
$$

which is evaluated numerically using the trapezoid rule. The mean-field models predict the mean location of the agents reasonably accurately for a range of bias strengths. Fig. 4 also shows the mean location for a population of agents moving without crowding effects (i.e. with no aborted movements), which is simply $v(0) t[19]$. This shows that crowding effects reduce the average distance moved in both the lattice-based and lattice-free models. We also note that agents in the lattice-based model move further than agents in the lattice-free model. This difference is because crowding effects are felt more strongly in the lattice-free model, and confirms that our approximation of the probability of a successful movement, $P(C)$, accurately captures these differences.

Since the lattice-based and lattice-free models involve different parameters to control the bias strength, the mean location results in Fig. 4 results are 


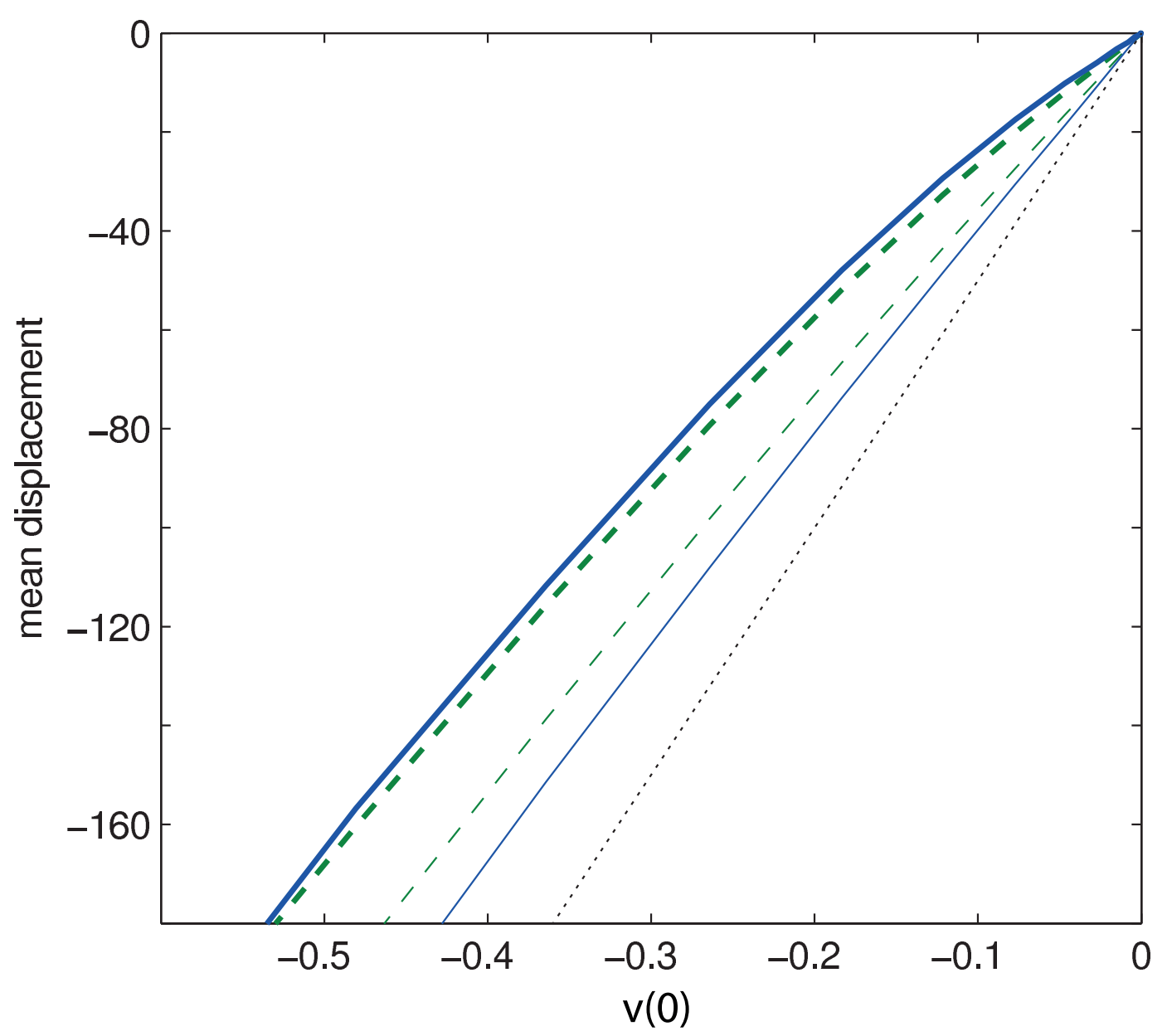

Fig. 4. Mean horizontal location at time $t=500$ averaged over $m=100$ realisations of the individual-based model (solid blue) and for the mean-field PDE (dashed green) against the low-density advection coefficient $v(0)$ for the lattice-based (thin) and lattice-free (thick) models. The dotted black line shows the mean horizontal location of a population moving without crowding effects, $v(0) t$. Parameter values: $P_{m}=1, \Delta=\tau=1$. Initial condition: $N=120$ agents randomly located in $-10<x<10,-5<y<5$.

given as a function of $v(0)$, which is a measure of bias strength applicable to both models; $v(0)$ is related to the bias parameters $\rho_{x}$ and $\sigma_{d}$ using Eqs. (4) and (6) respectively. In the lattice-based model, the restriction that $\left|\rho_{x}\right| \leq 1$ means that $|v(0)| \leq P_{m} \Delta /(2 \tau)$, whereas in the lattice-free model $\left|c_{1}\right| \leq 1$ so $|v(0)| \leq P_{m} \Delta / \tau$. Hence, for the same values of $\Delta$ and $\tau$, the lattice-free model can support stronger bias than the lattice-based model. This is a consequence of the standard assumption that the probabilities of moving vertically in the lattice-based model are fixed at $\left(1 \pm \rho_{y}\right) / 4$ in each vertical direction, so the maximum probability of moving the preferred direction is $1 / 2$. In the latticefree model, in the limit $\sigma_{d} \rightarrow 0$, the probability of moving in the preferred direction tends to 1 . 


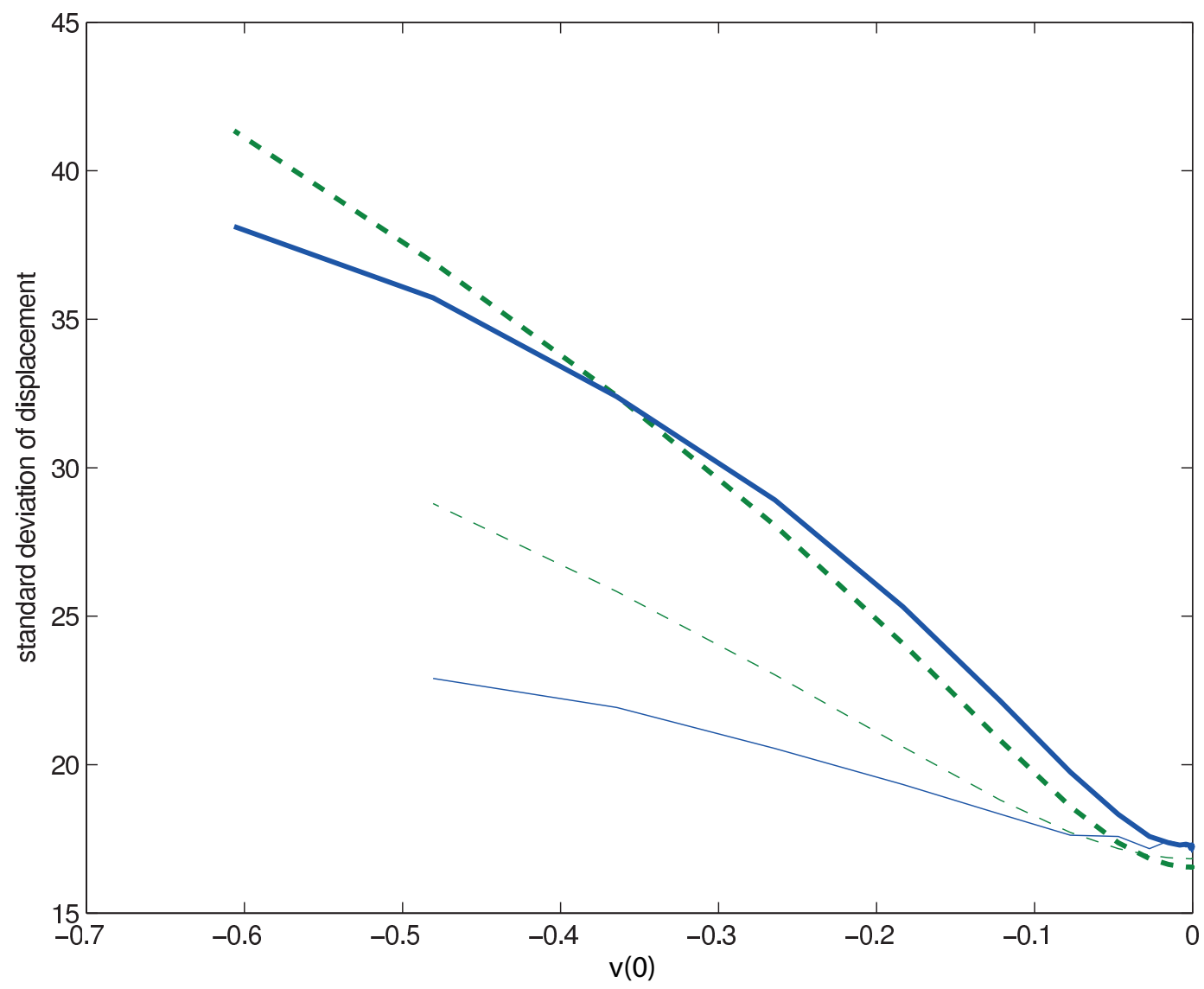

Fig. 5. Standard deviation of horizontal location at time $t=500$ in $m=100$ realisations of the individual-based model (solid blue) and for the mean-field PDE (dashed green) against the low-density advection coefficient $v(0)$ for the lattice-based (thin) and lattice-free (thick) models. The standard deviation of the horizontal location of a population moving without bias and without crowding effects is $s_{0}(t)=16.8$ at time $t=500$. Parameter values: $P_{m}=1, \Delta=\tau=1$. Initial condition: $N=120$ agents randomly located in $-10<x<10,-5<y<5$.

Fig. 5 shows the standard deviation of the horizontal location of all agents, starting from the same initial condition as in Fig. 3, at time $t=500$, as a culated analogously to Eq. (8) and the standard deviation is then calculated in the usual way. The results in Fig. 5 measure the amount of horizontal spreading of the population. The standard deviation of the horizontal location of a population of agents starting from the same initial condition and moving with neither bias nor crowding effects is $s_{0}(t)=\left(2 D(0) t+x_{0}^{2} / 3\right)^{1 / 2}[19]$. Crowding effects have little effect on the spreading in the unbiased case: all curves in Fig. 5 converge approximately on $s_{0}(t)$ at $v(0)=0$. As bias increases, crowding effects cause the population to become increasingly spread out (higher standard deviation). This is a reflection of the results seen in Fig. 3, where the leading edge of the biased population moves more rapidly than the trailing edge, leading to increased spreading. The mean-field PDE captures these ef- 
fects in the lattice-based model reasonably accurately for weak bias, but loses accuracy as the bias becomes stronger. Interestingly, the mean-field PDE for the lattice-free model provides a reasonable match even at very strong bias (stronger than is even possible in the lattice-based model).

\section{Discussion and Conclusion}

We have developed a lattice-free individual-based model for directed cell motion. This model is applicable in a variety of biological settings where cells are moving in the presence of a chemoattractant or chemorepellent. To provide mathematical insight into the discrete model, we derived an approximate mean-field description, which takes the form of a nonlinear advection-diffusion equation. Crowding effects in the mean-field description are represented by a function $P(C)$, which approximates the probability that an attempt to place an agent on a strip, at density $C$, is successful. Our model is different from classical random walk models, which ignore crowding effects, implicitly assuming $P(C)=1$, which leads to a linear advection-diffusion equation [19]. In lattice-based models with crowding effects, $P(C)$ is simply the probability of a randomly chosen lattice site being vacant, so that $P(C)=1-C$. This leads to an equation where the diffusion term is linear and the advection term is nonlinear [17]. Our recent results [45] gave an approximate expression for $P(C)$ in an unbiased lattice-free model, which led to a nonlinear diffusion term. Here, we have extended this framework to deal with biased motion, which leads to an advection-diffusion equation where both the advection and diffusion terms are nonlinear. Comparing averaged agent density profiles to the solution of our PDE description confirms that the mean-field PDE provides an accurate description of the average behaviour, thereby confirming that $P(C)$ captures the key crowding effects in the individual-based model.

Directional bias is modelled differently in the lattice-based and lattice-free models. In the lattice-based model, the probability that an isolated motile agent moves parallel to any given direction is constant. In other words, movement in the horizontal direction is independent of movement in the vertical direction. In contrast, the direction of movement in the lattice-free model is drawn from a continuous, circular distribution. As the bias becomes stronger, the probability of moving in the preferred direction increases at the expense of the probability of moving in a wider and wider range of other directions. This difference means that the lattice-free model can support stronger bias than the lattice-based model. It would be possible to modify the lattice-based model so that probabilities of choosing to move in the vertical direction could decrease as horizontal bias becomes stronger. One of the advantages of the lattice-free framework is that the preferred direction can be any angle $\theta \in[0,2 \pi]$ and the bias strength is controlled by a single parameter. We have investigated 
collective cell motion with a fixed direction of bias. This is relevant in some experimental scenarios, for example where cells are migrating towards a distant or a spatially extended source of chemoattractant. It would also be possible to introduce a spatial or temporal dependence into the direction of bias, for example to model the chemotactic response to a variable concentration gradient.

Dyson and Baker [30] considered a biased lattice-free model with volume exclusion. Bias was modelled by assigning a higher probability to movement directions within $\pi / 2$ of the preferred direction. Our model offers more flexibility in describing the effects of directional bias as the movement direction is drawn from a continuous circular distribution, which is the natural class of distribution for movement directions in a two-dimensional space [60]. Although we have used a wrapped normal distribution in the numerical results, this could readily be substituted by any other circular distribution, such as a von Mises distribution, a wrapped Cauchy distribution or even non-unimodal distributions. For example, if movement along a given axis were more likely than movement perpendicular to it, this could be modelled using a bimodal distribution peaked at directions parallel to the preferred axis (e.g. at $\theta=0$ and $\theta=\pi$ for a horizontal axis).

Our mean-field PDE model for the lattice-free model can be generalised to include descriptions of populations where multiple types, or species, of agents are present. The motion of each species can be governed by its own set of motility and bias parameters. In this more general case, the PDE for the average agent density of the $i^{\text {th }}$ species, $C_{i}(x, t)$, is

$$
\frac{\partial C_{i}}{\partial t}=-v_{i} \frac{\partial}{\partial x}\left(P(\bar{C}) C_{i}\right)-D_{i} \frac{\partial}{\partial x}\left(C_{i} \frac{\partial}{\partial x} P(\bar{C})\right)+D_{i} \frac{\partial}{\partial x}\left(P(\bar{C}) \frac{\partial C_{i}}{\partial x}\right),
$$

where $\bar{C}=\sum_{j} C_{j}$ is the total density of all species combined and $v_{i}$ and $D_{i}$ are the advection and diffusion coefficients respectively for species $i$ in the low-density limit. This generalises the lattice-based result of [17], where $P(C)=1-C$, to the lattice-free setting.

The continuum limit approximation we have derived gives a good prediction of average agent density when the bias is relatively weak, but breaks down if the bias is too strong. This is a common feature of biased random walk models where the bias affects the probability of moving in a given direction. Many continuum limit equations apply in a weak-bias limit $[19,23,63]$ and either break down or change form if the bias is sufficiently strong $[25,27,46]$. In contrast, models with background drift do not have this limitation as the drift induces a corresponding linear advection term in the continuum limit 
equation [31,32]. However, a background drift mechanism is more applicable as a model of convection, i.e. a deterministic component of agent movement, than of a directional bias in individual agents' movement probabilities. The continuum limit approximations derived by $[33,34]$ apply in the case without global bias and it is currently unknown whether these approaches will provide good approximations in the biased case.

The models considered in this study do not explicitly incorporate proliferation or death processes. We intentionally neglected these processes so that we could focus on directional bias in cell motility and we note that the neglect of proliferation is reasonable for typical experiments, which are conducted for time periods shorter than the cell doubling time [48]. Nevertheless, it would be straightforward to incorporate cell proliferation and death into the individualbased model. For the lattice-based case, this leads to a logistic growth term in the PDE [17] whereas, for the lattice-free model with unbiased motility, this leads to a different source term that is smaller than the logistic model [28]. It remains to be tested whether the lattice-free source term accurately captures proliferation in a lattice-free model that includes directional bias.

Previous work has shown that the choice of using a lattice-free or lattice-based model can affect estimates of cell proliferation rates from experimental density data in, for example, a growth-to-confluence experiment [12]. Constraining the cells to reside on an artificial lattice allows the population to reach confluence more rapidly and hence leads to reduced estimates of the intrinsic cell proliferation rate $[28,44]$. The results in the present study indicate that similar issues could arise when estimating motility parameters in the presence of a directional bias. For example, if experimental data describing the mean cell location were used to estimate the strength of bias, the results in Fig. 4 show that using a lattice-based model would lead to an underestimate of bias strength (smaller $|v(0)|$ ) compared to the lattice-free model.

As a final remark, we do not claim that the lattice-free model presented in this work is the correct description of directed cell motility, nor that the more standard approach based on a square lattice is incorrect. Instead, we aim to show that, when cells in the individual-based model are not restricted to reside and move on a predefined lattice, different outcomes are observed. Although we have shown results from a square lattice, other lattice geometries are possible and may give different results. Therefore, parameter estimates obtained by fitting standard, lattice-based models to experimental data should be treated with caution. 


\section{Acknowledgements}

500

This research was supported by RSNZ Marsden grant (11-UOC-005) and the Australian Research Council (FT130100148).

\section{References}

[1] A. R. A. Anderson, M. A. J. Chaplain, Continuous and discrete mathematical models of tumor-induced angiogenesis, Bulletin of Mathematical Biology 60 (5) (1998) 857-899.

[2] A. Q. Cai, K. A. Landman, B. D. Hughes, Multi-scale modeling of a woundhealing cell migration assay, Journal of Theoretical Biology 245 (3) (2007) 576594 .

[3] G. J. Pettet, M. A. J. Chaplain, D. L. S. McElwain, H. M. Byrne, On the role of angiogenesis in wound healing, Proceedings of the Royal Society of London. Series B: Biological Sciences 263 (1376) (1996) 1487-1493.

[4] D. Kaiser, Signaling in myxobacteria, Annual Review of Microbiology. 58 (2004) 75-98.

[5] M. Eisenbach, Chemotaxis, Wiley Online Library, 2004.

[6] J. D. Murray, Mathematical biology, Springer-Verlag, New York, 1989.

[7] E. Khain, M. Katakowski, S. Hopkins, A. Szalad, X. Zheng, F. Jiang, M. Chopp, Collective behavior of brain tumor cells: the role of hypoxia, Physical Review E 83 (3) (2011) 031920.

[8] A. Stevens, H. G. Othmer, Aggregation, blowup, and collapse: The abc's of taxis in reinforced random walks, SIAM Journal on Applied Mathematics 57 (4) (1997) 1044-1081.

[9] M. J. Simpson, A. Merrifield, K. A. Landman, B. D. Hughes, Simulating invasion with cellular automata: connecting cell-scale and population-scale properties, Physical Review E 76 (2) (2007) 021918.

[10] M. Ward, C. McCann, M. DeWulf, J. Y. Wu, Y. Rao, Distinguishing between directional guidance and motility regulation in neuronal migration, Journal of Neuroscience 23 (12) (2003) 5170-5177.

[11] P. K. Maini, D. L. S. McElwain, D. I. Leavesley, Traveling wave model to interpret a wound-healing cell migration assay for human peritoneal mesothelial cells, Tissue Engineering 10 (3-4) (2004) 475-482.

[12] A. Tremel, A. Q. Cai, N. Tirtaatmadja, B. D. Hughes, G. W. Stevens, K. A. Landman, A. J. O'Connor, Cell migration and proliferation during monolayer formation and wound healing, Chemical Engineering Science 64 (2) (2009) 247253. 
[13] T. M. Liggett, Stochastic Interacting Systems: Contact, Voter and Exclusion processes, Springer, Berlin, 1999.

[14] C. Deroulers, M. Aubert, M. Badoual, B. Grammaticos, Modeling tumor cell migration: from microscopic to macroscopic models, Physical Review E 79 (3) (2009) 031917.

[15] K. J. Painter, T. Hillen, Volume-filling and quorum-sensing in models for chemosensitive movement, Canadian Applied Mathematics Quarterly 10 (4) (2002) 501-543.

[16] L. M. Sander, T. S. Deisboeck, Growth patterns of microscopic brain tumors, Physical Review E 66 (5) (2002) 051901.

[17] M. J. Simpson, K. A. Landman, B. D. Hughes, Multi-species simple exclusion processes, Physica A 388 (4) (2009) 399-406.

[18] W. Alt, Biased random walk models for chemotaxis and related diffusion approximations, Journal of Mathematical Biology 9 (2) (1980) 147-177.

[19] E. A. Codling, M. J. Plank, S. Benhamou, Random walk models in biology, Journal of the Royal Society Interface 5 (25) (2008) 813-834.

[20] T. Callaghan, E. Khain, L. M. Sander, R. M. Ziff, A stochastic model for wound healing, Journal of Statistical Physics 122 (5) (2006) 909-924.

[21] E. Khain, L. M. Sander, C. M. Schneider-Mizell, The role of cell-cell adhesion in wound healing, Journal of Statistical Physics 128 (1-2) (2007) 209-218.

[22] M. J. Simpson, K. K. Treloar, B. J. Binder, P. Haridas, K. J. Manton, D. I. Leavesley, D. L. S. McElwain, R. E. Baker, Quantifying the roles of cell motility and cell proliferation in a circular barrier assay, Journal of The Royal Society Interface 10 (82) (2013) 20130007.

[23] H. G. Othmer, S. R. Dunbar, W. Alt, Models of dispersal in biological systems, Journal of Mathematical Biology 26 (3) (1988) 263-298.

[24] M. J. Plank, B. D. Sleeman, Lattice and non-lattice models of tumour angiogenesis, Bulletin of Mathematical Biology 66 (6) (2004) 1785-1819.

[25] E. A. Codling, R. N. Bearon, G. J. Thorn, Diffusion about the mean drift location in a biased random walk, Ecology 91 (10) (2010) 3106-3113.

[26] T. Hillen, H. G. Othmer, The diffusion limit of transport equations derived from velocity-jump processes, SIAM Journal on Applied Mathematics 61 (3) (2000) $751-775$.

[27] H. G. Othmer, T. Hillen, The diffusion limit of transport equations II: Chemotaxis equations, SIAM Journal on Applied Mathematics 62 (4) (2002) $1222-1250$.

[28] M. J. Plank, M. J. Simpson, Models of collective cell behaviour with crowding effects: comparing lattice-based and lattice-free approaches, Journal of The Royal Society Interface 9 (76) (2012) 2983-2996. 
29] L. Dyson, P. K. Maini, R. E. Baker, Macroscopic limits of individual-based models for motile cell populations with volume exclusion, Physical Review E 86 (3) (2012) 031903.

[30] L. Dyson, R. E. Baker, The importance of volume exclusion in modelling cellular migration, Journal of mathematical biology (2014) 1-21.

[31] M. Bruna, S. J. Chapman, Excluded-volume effects in the diffusion of hard spheres, Physical Review E 85 (1) (2012) 011103.

[32] M. Bruna, S. J. Chapman, Diffusion of multiple species with excluded-volume effects, Journal of Chemical Physics 137 (20) (2012) 204116.

[33] A. M. Middleton, C. Fleck, R. Grima, A continuum approximation to an offlattice individual-cell based model of cell migration and adhesion, Journal of Theoretical Biology 339 (2014) 220-232.

[34] R. N. Binny, M. J. Plank, A. James, Spatial moment dynamics for collective cell movement incorporating a neighbour-dependent directional bias, Journal of The Royal Society Interface 12 (106) (2015) 20150228.

[35] M. J. Simpson, K. A. Landman, B. D. Hughes, Cell invasion with proliferation mechanisms motivated by time-lapse data, Physica A 389 (18) (2010) 37793790.

[36] R. A. Fisher, The wave of advance of advantageous genes, Annals of Eugenics 7 (4) (1937) 355-369.

[37] A. N. Kolmogorov, I. G. Petrovsky, N. S. Piskunov, Étude de l'équation de la diffusion avec croissance de la quantité de matière et son application à un problème biologique, Moscow University Bulletin of Mathematics 1 (1937) 1-25.

[38] K. Anguige, C. Schmeiser, A one-dimensional model of cell diffusion and aggregation, incorporating volume filling and cell-to-cell adhesion, Journal of Mathematical Biology 58 (3) (2009) 395-427.

[39] C. J. Penington, B. D. Hughes, K. A. Landman, Building macroscale models from microscale probabilistic models: a general probabilistic approach for nonlinear diffusion and multispecies phenomena, Physical Review E 84 (4) (2011) 041120.

[40] N. J. Armstrong, K. J. Painter, J. A. Sherratt, A continuum approach to modelling cell-cell adhesion, Journal of Theoretical Biology 243 (1) (2006) 98113.

[41] E. Khain, L. M. Sander, Generalized cahn-hilliard equation for biological applications, Physical Review E 77 (5) (2008) 051129.

[42] V. Cristini, X. Li, J. S. Lowengrub, S. M. Wise, Nonlinear simulations of solid tumor growth using a mixture model: invasion and branching, Journal of Mathematical Biology 58 (4-5) (2009) 723-763. 
[43] A. E. Fernando, K. A. Landman, M. J. Simpson, Nonlinear diffusion and exclusion processes with contact interactions, Physical Review E 81 (1) (2010) 011903.

[44] M. J. Plank, M. J. Simpson, Lattice-free models of cell invasion: discrete simulations and travelling waves, Bulletin of Mathematical Biology 75 (11) (2013) 2150-2166.

[45] S. T. Johnston, M. J. Simpson, M. J. Plank, Lattice-free descriptions of collective motion with crowding and adhesion, Physical Review E 88 (6) (2013) 062720 .

[46] A. Cheung, S. Zhang, C. Stricker, M. V. Srinivasan, Animal navigation: the difficulty of moving in a straight line, Biological cybernetics 97 (1) (2007) 4761.

[47] N. A. Hill, D.-P. Häder, A biased random walk model for the trajectories of swimming micro-organisms, Journal of Theoretical Biology 186 (4) (1997) 503526.

[48] M. J. Simpson, K. K. Treloar, B. J. Binder, P. Haridas, K. J. Manton, D. I. Leavesley, D. L. S. McElwain, R. E. Baker, Quantifying the roles of cell motility and cell proliferation in a circular barrier assay, Journal of The Royal Society Interface 10 (2013) 20130007.

[49] M. Aubert, M. Badoual, S. Fereol, C. Christov, B. Grammaticos, A cellular automaton model for the migration of glioma cells, Physical Biology 3 (2) (2006) 93.

[50] M. Aubert, M. Badoual, B. Grammaticos, A model for short-and long-range interactions of migrating tumour cell, Acta Biotheoretica 56 (4) (2008) 297314 .

[51] C. A. Yates, R. E. Baker, Importance of the voronoi domain partition for position-jump reaction-diffusion processes on nonuniform rectilinear lattices, Physical Review E 88 (5) (2013) 054701.

[52] T. Alarcón, H. M. Byrne, P. K. Maini, A cellular automaton model for tumour growth in inhomogeneous environment, Journal of Theoretical Biology 225 (2) (2003) 257-274.

[53] B. J. Binder, K. A. Landman, Exclusion processes on a growing domain, Journal of Theoretical Biology 259 (3) (2009) 541-551.

[54] S. R. McDougall, M. G. Watson, A. H. Devlin, C. A. Mitchell, M. A. J. Chaplain, A hybrid discrete-continuum mathematical model of pattern prediction in the developing retinal vasculature, Bulletin of Mathematical Biology 74 (10) (2012) 2272-2314.

[55] G. P. Figueredo, T. V. Joshi, J. M. Osborne, H. M. Byrne, M. R. Owen, Onlattice agent-based simulation of populations of cells, Interface Focus 2013. 
[56] B. L. Cheeseman, D. Zhang, B. J. Binder, D. F. Newgreen, K. A. Landman, Cell lineage tracing in the developing enteric nervous system: superstars revealed by experiment and simulation, Journal of The Royal Society Interface 11 (93) (2014) 20130815.

[57] P. Strating, Brownian dynamics simulation of a hard-sphere suspension, Physical Review E 59 (2) (1999) 2175.

[58] B. J. Brosilow, R. M. Ziff, R. D. Vigil, Random sequential adsorption of parallel squares, Physical Review A 43 (2) (1991) 631.

[59] E. L. Hinrichsen, J. Feder, T. Jøssang, Geometry of random sequential adsorption, Journal of statistical physics 44 (5-6) (1986) 793-827.

[60] K. V. Mardia, P. E. Jupp, Directional statistics, John Wiley \& Sons, 2009.

${ }_{662}$ [61] R. E. Baker, M. J. Simpson, Correcting mean-field approximations for birthdeath-movement processes, Physical Review E 82 (4) (2010) 041905.

[62] S. T. Johnston, M. J. Simpson, R. E. Baker, Mean-field descriptions of collective migration with strong adhesion, Physical Review E 85 (5) (2012) 051922.

[63] B. D. Hughes, Random Walks and Random Environments. Volume 1: Random Walks, Clarendon Press, Oxford, 1995.

[64] S. C. Chapra, R. P. Canale, Numerical methods for engineers, McGraw-Hill, New York, 1998.

[65] L. F. Shampine, M. W. Reichelt, The Matlab ODE suite, SIAM Journal on Scientific Computing 18 (1) (1997) 1-22. 